\title{
Das Schutzlandprinzip als Grundlage für die Behandlung von grenzüberschreitenden Schadensersatzansprüchen aus der Verletzung geistigen Eigentums
}

\author{
Simon Laimer
}

\section{Einleitung}

Weist ein immaterialgüterrechtlicher Sachverhalt grenzüberschreitende Bezüge, mithin Verbindungen zu mehr als einer Rechtsordnung auf, dann ist das darauf anzuwendende nationale Recht nach dem Internationalen Privatrecht des angerufenen Gerichts zu ermitteln. ${ }^{1}$ Die demnach vorgelagerte ${ }^{2}$ Problematik der internationalen gerichtlichen Zuständigkeit wird bereits an anderer Stelle in diesem Band ${ }^{3}$ vertieft und daher hier nicht behandelt. Vom Immaterialgüterrechtsstatut, mithin dem Recht, das den Bestand, Inhalt und Schutz von geistigen Eigentumsrechten ${ }^{4}$ regelt, ist allerdings das - aus der Sicht EU-mitgliedstaatlicher Gerichte von der

\footnotetext{
${ }^{1}$ Siehe auch Grünberger, Das Urheberrechtsstatut nach der Rom II-VO, ZVglRWiss 108 (2009) 134; Jegher/Vasella, in: Basler Kommentar - Internationales Privatrecht ${ }^{3}$ (2013) Art. 110 Rn. 1.

${ }^{2}$ Mankowski, Internationale Zuständigkeit und anwendbares Recht - Parallelen und Divergenzen, FS Heldrich (2005) $867 \mathrm{ff}$.

${ }^{3}$ Siehe Kindler, Der europäische Deliktsgerichtsstand und die gewerblichen Schutzrechte, GRUR 2018, 1107, und in diesem Band Kap. 7. Zur internationalen Zuständigkeit schweizerischer Gerichte Kernen, Persönlichkeitsverletzungen im Internet (2014) 65 ff.

${ }^{4}$ Zur Problematik des im deutschen Sprachgebrauch (auch in der deutschsprachigen Fassung des Art. 8 Abs. 1 Rom II-VO; zur VO unten Fn. 17) alternativ zum Begriff „Immaterialgüterrecht“ verwendeten Ausdruck ,geistiges Eigentum“ siehe Götting, Der Begriff des Geistigen Eigentums, GRUR 2006, 353.
}

\footnotetext{
S. Laimer $(\bowtie)$

Institut für Zivilrecht, Johannes Kepler Universität Linz, Linz, Österreich

E-Mail: simon.laimer@jku.at
} 
Rom I-Verordnung, ${ }^{5}$ in der Schweiz von Art. 122 IPRG 6 erfasste - Statut immaterialgüterrechtlicher Verträge zu unterscheiden, ${ }^{7}$ das die schuldrechtlichen Beziehungen zwischen den Vertragsparteien beherrscht. ${ }^{8}$

In diesem Beitrag, der das Unions-IPR in den Mittelpunkt stellt und einen vergleichenden Blick auf das schweizerische Kollisionsrecht wirft, soll es allein um die kollisionsrechtliche Anknüpfung von außervertraglichen Schuldverhältnissen gehen, die aus einer Verletzung von Rechten des geistigen Eigentums entstehen (Schutzstatut). Auch für die vorauszuschickenden Fragen nach Entstehung, Bestand und Erlöschen von Immaterialgüterrechten (Bestandsstatut) ${ }^{9}$ sind nach in Deutschland und Österreich herrschender Meinung ${ }^{10}$ freilich dieselben Anknüpfungsgesichtskriterien heranzuziehen, ${ }^{11}$ sodass einer Unterscheidung zwischen Bestandsund Schutzstatut für die Zwecke dieses Beitrags keine wesentliche Bedeutung zukommt. ${ }^{12}$ In der Schweiz wird von einer umfassenden Reichweite des Immaterialgüterstatuts nach Art. 110 Abs. 1 IPRG ausgegangen. ${ }^{13}$ Vergleichsweise ist in Liechtenstein ausdrücklich das „Entstehen, der Inhalt und das Erlöschen von

\footnotetext{
${ }^{5}$ Die Verordnung (EG) 593/2008 v. 17.06.2008 über das auf vertragliche Schuldverhältnisse anzuwendende Recht („Rom I“), ABl 2008 L 177/6, berichtigt in ABl 2009 L 309/87, gilt in den EU-Mitgliedstaaten - mit Ausnahme von Dänemark - als einheitliches Kollisionsrecht für ab dem 17.12.2009 geschlossene vertragliche Schuldverhältnisse.

${ }^{6}$ (Schweizerisches) Bundesgesetz v. 18.12.1987 über das Internationale Privatrecht (SR 291).

${ }^{7}$ Fezer/Koos, in: J. von Staudingers Kommentar zum Bürgerlichen Gesetzbuch. Internationales Wirtschaftsrecht (2019) Rn. 927 f., 968 ff.

${ }^{8}$ Vgl. etwa Stimmel, Die Beurteilung von Lizenzverträgen unter der Rom I-Verordnung, GRUR Int 2010, $783 \mathrm{ff}$.

${ }^{9}$ Siehe hierzu Leible/Lehmann, Die neue EG-Verordnung über das auf außervertragliche Schuldverhältnisse anzuwendende Recht („Rom II“), RIW 2007, 721 (731); Heiss/Loacker, Die Vergemeinschaftung des Kollisionsrechts der außervertraglichen Schuldverhältnisse durch Rom II, JB1 2007, 613 (636).

${ }^{10}$ Drexl, in: Münchener Kommentar zum Bürgerlichen Gesetzbuch. Bd. 12: Internationales Privatrecht II, Internationales Wirtschaftsrecht, Einführungsgesetz zum Bürgerlichen Gesetzbuche, 7. Aufl. (2018) Rn. 199; Spickhoff, in: Beck'scher Online-Kommentar zum BGB, 53. Ed. 2020, VO (EG) 864/2007 Art. 8 Rn. 5 m.w.N.; anders Schack, Das auf (formlose) Immaterialgüterrechte anwendbare Recht nach Rom II, FS Kropholler (2008) 651 (653).

${ }^{11}$ Zur Anknüpfung nach Art. 34 AT-IPRG (der eine Gesamtverweisung normiert, vgl. OGH 4 Ob 184/13g, GRUR Int. 2014, 697, mit Anm. von Walter = ÖB1 2014, 134, mit Anm. von Büchele) siehe Lurger/Melcher, Handbuch Internationales Privatrecht (2017) Rn. 5/80. Zur Anknüpfung nach dem deutschen autonomen Kollisionsrecht Wagner, Die neue Rom-II-Verordnung, IPRax 2008, 1 (9); v. Hein, Europäisches Internationales Deliktsrecht nach der Rom II-Verordnung, ZEuP 2009, 6 (30); Mankowski, Besondere Formen von Wettbewerbsverstößen im Internet und Internationales Wettbewerbsrecht, GRUR Int. 1999, 995 (996 f.); BGHZ 155, 257 (261); 141, 267 (277 ff.); 136, 380 (387). Zur - für einen renvoi bedeutsamen - Anknüpfung im IPR mancher Staaten an die lex originis siehe jedoch Mankowski, Internationales Privatrecht. Bd. II: Besonderer Teil $^{2}$ (2019) § 2 Rn. 357 m.w.N.

${ }^{12}$ Staudinger/Fezer/Koos, BGB, IntWirtschR Rn. 867.

${ }^{13}$ Jegher/Vasella, in: BaK ${ }^{3}$ Art. 110 Rn. 9.
} 
Immaterialgüterrechten" von dem - auf das Vorbild des Art. 34 Abs. 1 AT-IPRG ${ }^{14}$ zurückgehenden - Art. 38 Abs. 1 FL-IPRG ${ }^{15}$ erfasst. $^{16}$

Die hier im Mittelpunkt stehenden Folgen einer - ab dem 11.01.2009 vorgenommenen (Art. 31 f. Rom II-VO) $)^{17}$ - Verletzung geistiger Eigentumsrechte, ${ }^{18}$ mithin der aus dem Eingriff resultierende Anspruch auf Schadensersatz, aber auch etwa die Ansprüche auf Auskunft, Unterlassung, Beseitigung oder Gewinnherausgabe, werden im Sinne der Erweiterung des Anwendungsbereichs gem. Art. 13 Rom II-VO von der Sonderanknüpfungsnorm des Art. 8 Rom II-VO (in der Schweiz von Art. 110 IPRG) ${ }^{19}$ erfasst, ${ }^{20}$ womit eine einheitliche Qualifikation der bei Verletzung geistigen Eigentums entstehenden Ansprüche sichergestellt ist. ${ }^{21}$ Im Unterschied zum Lauterkeitskollisionsrecht, wo Art. 6 Abs. 1 Rom II-VO lediglich eine Präzisierung des Erfolgsortes der allgemeinen Deliktskollisionsnorm nach Art. 4 Abs. 1 Rom II-VO darstellt, ${ }^{22}$ handelt es sich bei Art. 8 Rom II-VO um eine echte Sonderanknüpfung. ${ }^{23}$ Das danach bestimmte Recht ist für die Anspruchsvoraussetzungen, die Haftungsausschlussgründe, die Bestimmung der haftenden Personen ${ }^{24}$ und des anspruchsberechtigten Personenkreises sowie für die Rechtsfolgen maßgeblich (vgl. Art. 15 Rom II-VO). ${ }^{25}$

\footnotetext{
${ }^{14}$ (Liechtensteinisches) Gesetz v. 19.09.1996 über das internationale Privatrecht, LGB1. 1996/194 (LR-Nr. 290).

${ }^{15}$ (Österreichisches) Bundesgesetz v. 15.06.1978 über das internationale Privatrecht, BGB1. 304/1978.
}

${ }^{16} \mathrm{Zu}$ Art. 54 IT-IPRG (ital. Gesetz v. 31.05.1995 Nr. 218) siehe hingegen Eccher, in: Christandl/ Eccher/Gallmetzer/Laimer/Schurr, Handbuch Italienisches Internationales Privatrecht (2019) Rn. $7 / 22 \mathrm{ff}$.

${ }^{17}$ Verordnung (EG) 864/2007 v. 11.07.2007 über das auf außervertragliche Schuldverhältnisse anzuwendende Recht (,Rom II“), ABl 2007 L 199/40.

${ }^{18}$ Dabei ist der Begriff „Verletzung“ weit zu verstehen, so dass alle Eingriffshandlungen erfasst sind, die rechtlich exklusiv dem Rechtsinhaber zugewiesen wären, Mankowski, IPR II: BT ${ }^{2}$ (2019) $\S 2$ Rn. 355.

${ }^{19}$ In Liechtenstein gilt hierfür Art. 38 FL-IPRG, dazu etwa FL-OGH, GRUR Int 1980, 529 (530); GRUR Int 1998, 512 (515).

${ }^{20}$ Metzger, Geistiges Eigentum (Kollisionsrecht), in: Basedow/Hopt/Zimmermann (Hrsg.), Handwörterbuch des Europäischen Privatrechts (2009) 661 (663); Neumayr, in: Koziol/Bydlinski/Bollenberger (Hrsg.), ABGB ${ }^{6}$ (2020) Art. 8 Rom II-VO Rn. 1. Zur Vorgeschichte ausführlich Basedow/Metzger, in: Trunk/Knieper/Svetlanov (Hrsg.), Russland im Kontext der internationalen Entwicklung: Internationales Privatrecht, Kulturgüterschutz, geistiges Eigentum, Rechtsvereinheitlichung (2004) 153.

${ }^{21}$ Dörner, in: Schulze u.a., Bürgerliches Gesetzbuch - Handkommentar ${ }^{10}$ (2019) Rom II Art. 13 Rn. 1; Neumayr, in: $\mathrm{KBB}^{6}$ Art. 8 Rom II-VO Rn. 2.

${ }^{22}$ ErwGr. 21 S. 1 Rom II-VO.

${ }^{23}$ Sack, Das IPR des geistigen Eigentums nach der Rom II-VO, WRP 2008, 1405 (1413).

${ }^{24}$ Zur Haftung mittelbarer Verletzer, mithin „Intermediäre“ wie z. B. Vertreiber von File-Sharing-Software, siehe etwa de la Durantaye, in: Callies (ed.), Rome Regulations: Commentary ${ }^{2}$ (2015) Rome II Art. 8 Rn. 23 f.

${ }^{25}$ Staudinger/Fezer/Koos, BGB, IntWirtschR Rn. 876. Die Inhaberschaft an einem IP-Recht ist jedoch eine Vorfrage (str.), Mankowski, IPR II: BT ${ }^{2}$ (2019) § 2 Rn. 358 f. m. w. N. 
Der ausschließlich ${ }^{26}$ und universell (Art. 3 Rom II-VO) ${ }^{27}$ anzuwendende Art. 8 Rom II-VO verdrängt in seinem sachlichen und zeitlichen Anwendungsbereich die nationalen Kollisionsrechte der EU-Mitgliedstaaten mit Ausnahme Dänemarks. ${ }^{28}$ Selbst internationale Übereinkommen sind allein dann vorrangig heranzuziehen, wenn auch Drittstaaten daran beteiligt sind (Art. 28 Abs. 1 Rom II-VO). ${ }^{29}$ Der originär kollisionsrechtliche Gehalt des hier einschlägigen Konventionsrechts, insbesondere der Grundsatz der Inländerbehandlung, auf den noch zurückzukommen sein wird (unten III.1.), ist jedoch umstritten. ${ }^{30}$ Internationalprivatrechtliche Fragen treten freilich auch im Zusammenhang mit den später noch anzusprechenden einheitlichen Unionsschutzrechten auf (unten III.2.). Im Folgenden ist freilich zunächst zu definieren, wann von ,geistigem Eigentum“ i. S. d. Art. 8 Rom II-VO (bzw. in der Schweiz von „Immaterialgüterrecht“ i. S. v. Art. 110 IPRG) gesprochen werden kann (II.). Anschließend werden die spezifischen Anknüpfungsgrundsätze im Überblick behandelt (III.). Ein abschließendes Fazit (IV.) rundet den Beitrag ab.

\section{Verweisungsgegenstand: Recht des geistigen Eigentums}

Der unionsrechtsautonom auszulegende ${ }^{31}$ Begriff ,geistiges Eigentum“ in Art. 8 Abs. 1 Rom II-VO umfasst beispielsweise Urheberrechte, ${ }^{32}$ verwandte Schutzrechte, das Schutzrecht sui generis für Datenbanken sowie gewerbliche

\footnotetext{
${ }^{26}$ Siehe dazu auch unten bei 3.1. Auf dem Gebiet einschlägige Unionsrechtsakte enthalten jedoch teilweise gem. Art. 27 Rom II-VO vorrangige Sonderkollisionsnormen, MacGuire, in: beck-online.Großkommentar (2017) Art. 8 Rom II-VO Rn. 180; Grubinger, Die Rom II-VO und die Verletzung von freiem Wettbewerb sowie von Rechten des geistigen Eigentums, in: Beig u.a. Rom II-VO. Neues Kollisionsrecht für außervertragliche Schuldverhältnisse (2008) 55 (64 f.); anders Grünberger, in: Hüßtege/Mansel (Hrsg.), NomosKommenar BGB, Bd. 6: Rom-Verordnungen ${ }^{3}$ (2019) Art. 8 Rom II-VO Rn. 19.

${ }^{27} \mathrm{MüKoBGB} /$ Drexl, IntImmGR Rn. 161.

${ }^{28}$ ErwGr. 40 Rom II-VO. Zum durchaus noch beachtlichen verbleibenden Anwendungsbereich des nationalen Kollisionsrechts MüKoBGB/Drexl, IntImmGR Rn. 192 ff.

${ }^{29}$ Siehe insbes. Pariser Verbandsübereinkunft zum Schutze des gewerblichen Eigentums (PVÜ) v. 20.03.1883; Madrider Abkommen über die internationale Registrierung von Marken (MMA) v. 14.04.1891 samt Protokoll v. 27.6.1989; Abkommen über handelsbezogene Aspekte der Rechte des geistigen Eigentums (TRIPS) v. 14.04.1994; Revidierte Berner Übereinkunft zum Schutz von Werken der Literatur und Kunst (RBÜ) v. 9.9.1886; Welturheberrechtsabkommen (WUA) v. 06.09.1952; Abkommen über den Schutz der ausübenden Künstler, Hersteller von Tonträgern und Sendeunternehmen (Rom-Abkommen) v. 26.10.1961; Europäisches Abkommen zum Schutz von Fernsehsendungen v. 22.06.1960; Internationales Übereinkommen zum Schutz von Pflanzenzüchtungen v. 02.12.1961; Münchener Übereinkommen über die Erteilung europäischer Patente v. 05.10.1973.

${ }^{30}$ Staudinger/Fezer/Koos, BGB, IntWirtschR Rn. $879 \mathrm{f}$.

${ }^{31}$ Grünberger, ZVglRWiss 108 (2009) 134 (136 f.).

${ }^{32}$ Siehe zum materiellen Urheberrecht in diesem Band Büchele, Aktuelle Rechtsprechung zum österreichischen und europäischen Urheberrecht, Kap. 5.
} 
Schutzrechte, ${ }^{33}$ mithin Rechte aus einem Patent, ${ }^{34}$ Geschmacks- und Gebrauchsmuster, ${ }^{35}$ aus dem Sorten- und Topografieschutz von Halbleitern, aus der Marke ${ }^{36}$ und den geschäftlichen Bezeichnungen. ${ }^{37}$

In diesem Sinne greift Art. 8 Rom II-VO auch beim gewerblich bzw. geschäftlich genutzten Internetdomain-Namen. ${ }^{38}$ Beinhaltet der Domain-Name indes einen privaten Namen, sodass er sich als Ausprägung des Allgemeinen Persönlichkeitsrechts erweist, dann ist die Bestimmung hingegen wegen der Ausnahmeregelung in Art. 1 Abs. 2 lit. g Rom II-VO für Rechtsverhältnisse aus Persönlichkeitsrechtsverletzung nicht einschlägig. ${ }^{39}$ Aufgrund der engen Verbindung zum Urhebernutzungsrecht $t^{40}$ fällt weiterhin das Urheberpersönlichkeitsrecht wie auch das Erfinderpersönlichkeitsrecht ${ }^{41}$ unter Art. 8 Rom II-VO. ${ }^{42}$ Ferner sind etwa geografische Herkunftsangaben als geistiges Eigentum zu qualifizieren. ${ }^{43}$

\section{Anknüpfungsgrundsätze}

\subsection{Recht des Schutzlandes}

Das Immaterialgüterrecht ist grundlegend von der tradierten Vorstellung geprägt, dass Bestand, Inhalt und Wirkung des in Bezug auf das geistige Eigentumsrecht entstandenen Schutzrechts räumlich auf das Territorium des Staates beschränkt ist,

\footnotetext{
${ }^{33}$ So ErwGr. 26 S. 2 Rom II-VO. Vgl. etwa Neumayr, in: KBB ${ }^{6}$ Art. 8 Rom II-VO Rn. 1.

${ }^{34}$ Zum italienischen materiellen Recht siehe in diesem Band Ferretti/Zito, Introduzione al diritto brevettuale italiano, Kap. 2.

${ }^{35}$ Siehe zum materiellen Recht in diesem Band Steichele, Die Grundzüge des Designrechts in Deutschland, Kap. 4; Ferretti/Zito, Introduzione al design: uno sguardo d'insieme, Kap. 3.

${ }^{36}$ Zum materiellen Recht in diesem Band Perathoner, Das italienische, europäische und internationale Markenrecht und Markenprozessrecht, Kap. 1.

${ }^{37}$ Eine Binnendifferenzierung zwischen verschiedenen Rechten des geistigen Eigentums findet also nicht statt, Heiss/Loacker, JBl 2007, 613 (634); Grünberger, ZVglRWiss 108 (2009) 134 (141). Vgl. OGH 4 Ob 12/11k, GRUR Int 2012, $468=$ RdW 2012, 218; 17 Ob 6/11y, GRUR Int 2012, 464 = RdW 2012, 154.

${ }^{38}$ BeckOK BGB/Spickhoff, VO (EG) 864/2007 Art. 8 Rn. 2.

${ }^{39}$ Weller, LMK 2013, 344766, zu BGH GRUR 2013, 294. Zu Unternehmenspersönlichkeitsrechten siehe allerdings R. Magnus, Unternehmenspersönlichkeitsrechte im digitalen Raum und Internationales Privatrecht, RabelsZ 84 (2020) $1 \mathrm{ff}$.

${ }^{40}$ So Staudinger/Fezer/Koos, BGB, IntWirtschR Rn. 877.

${ }^{41}$ OGH GRUR Int 2012, 468.

${ }^{42}$ BeckOK BGB/Spickhoff, VO (EG) 864/2007 Art. 8 Rn. 2.

${ }^{43}$ Sack, Das IPR des geistigen Eigentums nach der Rom II-VO, WRP 2008, 1405 (1046 f.); Staudinger/Fezer/Koos, BGB, IntWirtschR Rn. 877. Zur Abgrenzung vom - nach Art. 6 Rom II-VO bzw. in der Schweiz nach Art. 136 IPRG anzuknüpfenden - Lauterkeitsrecht siehe MüKoBGB/Drexl, IntImmGR Rn. 3 f.; Vischer, in: Zürcher Kommentar zum IPRG, 2. Aufl., 2004, Art. 136 Rn. 8.
} 
der es verleiht oder anerkennt. ${ }^{44}$ Dieses Territorialitätsprinzip wird auch von den internationalen Konventionen im Bereich des Immaterialgüterrechts vorausgesetzt, nach denen die Gleichstellung der Ausländer mit den Inländern in den verschiedenen nationalen Gebieten zu gewährleisten ist (Inländerbehandlungsgrundsatz). ${ }^{45}$ Während die Gegenauffassung das Universalitätsprinzip vertritt, wonach speziell ein weltweit anerkanntes einheitliches Urheberrecht existiere, das sich nach dem Ursprungsland richte (lex originis) ${ }^{46}$ gilt der Territorialitätsgrundsatz nach der herrschenden Auffassung auch für das Urheberrecht. ${ }^{47}$ Der territoriale Charakter des Urheberrechts wurde ferner vom Europäischen Gerichtshof in einer den grenzüberschreitenden Rundfunk betreffenden Entscheidung aus dem Jahr 2005 als unionsrechtliches Prinzip anerkannt. ${ }^{48}$

Vor dem Hintergrund des Territorialgrundsatzes im materiellen Recht, ${ }^{49}$ werden die Rechtsfragen, die sich bei der Erteilung und Wahrnehmung geistiger Eigentumsrechte ergeben, traditionell nach dem Recht des Schutz gewährenden Staates, der lex loci protectionis behandelt (Schutzlandprinzip).$^{50}$ In Art. 8 Abs. 1 Rom II-VO (und - weitergehend - in der Schweiz nach Art. 110 IPRG) ${ }^{51}$ ist die Schutzlandanknüpfung im Zusammenhang mit außervertraglichen Schuldverhältnissen (Delikt, Bereicherung, Geschäftsführung ohne Auftrag) verankert. ${ }^{52}$ Es kommt danach auf das Recht des Staates an, ,für den“, mithin für dessen Gebiet der Verletzte den Schutz des Immaterialgüterrechts beansprucht. ${ }^{53}$ Damit entspricht das Schutzlandrecht allein dann dem Recht des angerufenen Gerichts (lex fori), wenn das Schutzland mit dem Gerichtsstaat identisch ist. ${ }^{54}$

\footnotetext{
${ }^{44}$ MüKoBGB/Drexl, IntImmGR Rn. 161; Staudinger/Fezer/Koos, BGB, IntWirtschR Rn. 883; Troller, Das internationale Privat- und Zivilprozessrecht im gewerblichen Rechtsschutz und Urheberrecht (1952) 48; Metzger, in: Basedow/Hopt/Zimmermann (Hrsg.), Handwörterbuch, 661. Vgl. etwa BGE 126 III 129, 134.

${ }^{45}$ Grünberger, ZVglRWiss 108 (2009) 134 (146).

${ }^{46}$ Siehe Klass, Das Urheberkollisionsrecht der ersten Inhaberschaft - Plädoyer für einen universalen Ansatz, GRUR Int. 2007, 373 (380 ff.); Schack, Anknüpfung des Urheberrechts im internationalen Privatrecht (1979) Rn. 66; Drobnig, Originärer Erwerb und Übertragung von Immaterialgüterrechten im Kollisionsrecht, RabelsZ 40 (1976) 195 (196 ff.).

${ }^{47}$ Neumayr, in: KBB ${ }^{6}$ Art. 8 Rom II-VO Rn. 1; Staudinger/Fezer/Koos, BGB, IntWirtschR Rn. 885, $1064 \mathrm{ff}$.

${ }^{48}$ EuGH 14.07.2005, Rs. C-192/04, Lagardère Active Broadcast, ECLI:EU:C:2005:475 (Rn. 46).

${ }^{49}$ Manche entnehmen dem Territorialitätsprinzip bereits die kollisionsrechtliche Aussage, vgl. Vischer, in: ZK² Vor Art. 109-111 Rn. 6.

${ }^{50}$ Metzger, in: Basedow/Hopt/Zimmermann (Hrsg.), Handwörterbuch, 661

${ }^{51}$ BGer v. 07.08.2012, 4A_128/2012, E. 2; BGE 133 III 568, E. 3.

${ }^{52}$ Vgl. ErwGr. 26 S. 1 Rom II-VO. BeckOK BGB/Spickhoff, VO (EG) 864/2007 Art. 8 Rn. 4.

${ }^{53}$ Mankowski, IPR II: BT ${ }^{2}$ (2019) $§ 2$ Rn. 348; Staudinger/Fezer/Koos, BGB, IntWirtschR Rn. 910. Grundlegend Ulmer, Immaterialgüterrechte im internationalen Privatrecht (1975) 12. Vgl. OGH 17 Ob 6/11y, GRUR Int 2012, 464 = RdW 2012, 154.

${ }^{54}$ Vgl. OGH GUR Int. 1994, 638 (639).
} 
Erst nach dem Sachrecht des vom Kläger behaupteten Schutzstaates ist sodann $\mathrm{zu}$ untersuchen, ob eine verbotene Benutzungshandlung tatsächlich vorliegt. ${ }^{55} \mathrm{Be}$ hauptet der Anspruchssteller Verletzungshandlungen in mehreren Staaten, wie etwa bei Rechtsverletzungen im Internet ${ }^{56}$ dann ist an die Rechtsordnungen sämtlicher Schutzländer nebeneinander anzuknüpfen („Mosaikprinzip“) ${ }^{57}$ Dabei ist die Verweisung des Art. 8 Abs. 1 Rom II-VO (wie auch jene des Art. 110 Abs. 1 IPRG) ${ }^{58}$ als Sachnormverweisung auf das Schutzlandrecht zu betrachten. ${ }^{59}$

Die Sonderanknüpfung nach Art. 8 Abs. 1 Rom II-VO gilt ausschließlich, sodass in ihrem Anwendungsbereich weder eine parteiautonome Rechtswahl ${ }^{60}$ (Art. 8 Abs. 3 Rom II-VO; in der Schweiz ermöglicht Art. 110 Abs. 2 IPRG jedoch eine beschränkte Rechtswahl), ${ }^{61}$ noch eine akzessorische Anknüpfung an ein zwischen den Parteien bestehendes Rechtsverhältnis oder an den gemeinsamen gewöhnlichen Aufenthalt zulässig ist. ${ }^{62}$

\subsection{Unionsweit einheitliche Schutzrechte}

Ergänzend enthält Art. 8 Abs. 2 Rom II-VO eine Sonderbestimmung für einheitliche europäische Rechtstitel, die eine Unteranknüpfung an das Recht der Mitgliedstaaten ermöglichen soll, soweit die unionsrechtlichen Sekundärrechtsakte keine materiellrechtliche Regelung enthalten. ${ }^{63}$

Der Unionsgesetzgeber hat bisher mehrere solcher Systeme unionsweit geltender Immaterialgüterrechte geschaffen, die mit den auf nationaler Ebene fortbestehenden Schutzsystemen koexistieren: Dies betrifft die Unionsmarke, ${ }^{64}$ die gemeinsam mit dem Gemeinschaftsgeschmacksmuster ${ }^{65}$ durch das Amt der Europäischen

\footnotetext{
${ }^{55}$ MüKoBGB/Drexl, IntImmGR Rn. 12; Lurger, IZVR und IPR bei Internet Domain Namen, in: Mayer-Schönberger/Galla/Fallenböck (Hrsg.), Das Recht der Domain-Namen (2001) 103 (112 ff.). Ausführlich zum Schutzort Weller/Nordmeier, in: Spindler/Schuster, Recht der elektronischem Medien $^{3}$ (2015) Rom II Art. 8 Rn. 9 ff.

${ }^{56} \mathrm{Hk}-\mathrm{BGB} / \mathrm{Dörner}$, Rom II Art. 8 Rn. 4.

${ }^{57}$ Mankowski, IPR II: BT ${ }^{2}$ (2019) § 2 Rn. 351; Spindler, Die kollisionsrechtliche Behandlung von Urheberrechtsverletzungen im Internet, IPRax 2003, 412; Grünberger, ZVglRWiss 108 (2009) 134 (154). Vgl. OGH 4 Ob 190/12p, GRUR Int. 2013, 668 = RdW 2013, 394.

${ }^{58}$ Jegher/Vasella, in: BaK ${ }^{3}$ Art. 110 Rn. 25; BGE 136 III 232, E. 5.

${ }^{59}$ Staudinger/Fezer/Koos, BGB, IntWirtschR Rn. 922; Heiss/Loacker, JB1 2007, 635. Zu Eingriffsnormen und Ordre public-Vorbehalt MüKoBGB/Drexl, IntImmGR Rn. 253 ff.

${ }^{60}$ Krit. Leible, Rechtswahl im IPR der außervertraglichen Schuldverhältnisse nach der Rom II-Verordnung, RIW 2008, 257 (259); Schack, FS Kropholler, 656.

${ }^{61}$ Siehe hierzu unten bei 3.3 .

${ }^{62}$ Neumayr, in: KBB ${ }^{6}$ Art. 8 Rom II-VO Rn. 2, 5; BeckOK BGB/Spickhoff, VO (EG) 864/2007 Art. 8 Rn. 1.

${ }^{63}$ MüKoBGB/Drexl, IntImmGR Rn. 162.

${ }^{64}$ Verordnung (EU) 1001/2017 v. 14.06.2017 über die Unionsmarke, ABl. 2017 L 154/1.

${ }^{65}$ Verordnung (EG) 6/2002 v. 12.12.2001 über das Gemeinschaftsgeschmacksmuster, AB1. 2002 L 3/1.
} 
Union für das Geistige Eigentum (EUIPO) in Alicante erteilt wird, ${ }^{66}$ wobei unter gewissen Voraussetzungen auch ein nicht eingetragenes Gemeinschaftsgeschmacksmuster für die Dauer von drei Jahren geschützt wird. ${ }^{67}$ Ferner erteilt das Gemeinschaftssortenschutzamt in Angers das Gemeinschaftssortenschutzrecht. ${ }^{68}$ Geografische Herkunftsangaben ${ }^{69}$ trägt die Europäische Kommission in ein Register ein. Hinzu kommt ein durch das Europäische Patentamt in München einzutragendes Europäisches Patent mit einheitlicher Wirkung, ${ }^{70}$ deren nähere Ausgestaltung einem von den Staaten abzuschließenden Übereinkommen über ein einheitliches Patentgericht überlassen ist. Von diesen Unionsschutzrechten ist das Europäische Patentübereinkommen ${ }^{71} \mathrm{zu}$ unterscheiden, das die Anmeldung und Erteilung „Europäischer Patente" aufgrund eines einheitlichen Verfahrens ermöglicht, womit gleichwohl keine einheitliche Rechtsposition für das gesamte EU-Gebiet, sondern lediglich ein Bündel nationaler Patente begründet wird. ${ }^{72}$

Zunächst ist auch bei außervertraglichen Schuldverhältnissen aus der Verletzung solcher Unionsschutzrechte das Schutzland gem. Art. 8 Abs. 1 Rom II-VO zu eruieren (,Außen-IPR“) ${ }^{73}$ Wird der Schutz für das Gebiet der Europäischen Union beansprucht, so kommen die einschlägigen unionsrechtlichen Rechtsakte zur Anwendung. ${ }^{74}$ Die Union insgesamt ist hier als das maßgebliche Schutzland anzusehen. ${ }^{75}$ Soweit eine Regelung auf Unionsebene fehlt, wie vor allem in Bezug auf die Sanktionen bei Verletzung der einheitlichen Schutzrechte, dann ist ein Rückgriff auf das nationale Recht des Mitgliedstaates erforderlich, wofür das Schutzlandprinzip

\footnotetext{
${ }^{66}$ Knaak, Gemeinschaftsmarke, in: Basedow/Hopt/Zimmermann (Hrsg.), Handwörterbuch des Europäischen Privatrechts (2009) 676; Kur, Gemeinschaftsgeschmacksmuster, in: Basedow/Hopt/ Zimmermann (Hrsg.), Handwörterbuch des Europäischen Privatrechts (2009) 674.

${ }^{67}$ Siehe Art. 1 Abs. 2a der Gemeinschaftsgeschmacksmuster-VO.

${ }^{68}$ Verordnung (EG) $2100 / 1994$ v. 27.07.1994 über den gemeinschaftlichen Sortenschutz, AB1. 1994 L 258/3.

${ }^{69}$ Verordnung (EU) 1151/2012 v. 21.11.2012 über Qualitätsregelungen für Agrarerzeugnisse und Lebensmittel, AB1. 2012 L 343/1. Siehe in diesem Band auch Miribung, Die geografischen Angaben im CETA-Abkommen: Keine neuen Schläuche für alten Wein, Kap. 6.

${ }^{70}$ Verordnung (EU) 1257/2012 v. 17.12.2012 über die Umsetzung der Verstärkten Zusammenarbeit im Bereich der Schaffung eines einheitlichen Patentschutzes, ABl. 2012 L 361/1; Verordnung (EU) 1260/2012 v. 17.12.2012 über die Umsetzung der verstärkten Zusammenarbeit im Bereich der Schaffung eines einheitlichen Patentschutzes im Hinblick auf die anzuwendenden Übersetzungsregelungen, AB1. 2012 L 361/89. Hierzu Götting, Das EU-Einheitspatent: Das Ende einer ,unendlichen Geschichte"?, ZEuP 2014, 349; Ohly, Auf dem Weg zum Einheitspatent und zum Einheitlichen Patentgericht - Licht am Ende des Tunnels oder Tunnel am Ende des Lichts?, ZGE 4 (2012), 419.

${ }^{71}$ Europäisches Patentübereinkommen v. 05.10.1973, i. d. F. der Akte v. 29.11.2000 zur Revision des Übereinkommens, in der ab 13.12.2007 geltenden Fassung.

${ }^{72}$ MüKoBGB/Drexl, IntImmGR Rn. 37.

${ }^{73}$ Sack, WRP 2008, 1405 (1408 m. w. N.).

${ }^{74}$ Staudinger/Fezer/Koos, BGB, IntWirtschR Rn. 960.

${ }^{75}$ Metzger, in: Basedow/Hopt/Zimmermann (Hrsg.), Handwörterbuch, 661 (662).
} 
untauglich ist. ${ }^{76}$ Nach der Unteranknüpfung gem. Art. 8 Abs. 2 Rom II-VO ist in diesem Fall vielmehr das Recht des Staates anzuwenden, ,in dem die Verletzung begangen wurde“" ${ }^{77}$ mithin das Recht am Handlungsort (lex loci delicti commissi) (,Innen-IPR“). ${ }^{78}$

Bei Verletzungshandlungen in mehreren Staaten (z. B. über das Internet) könnte jedoch auch diese Regel eine Mosaikbetrachtung im Sinne einer Anwendung der Rechte sämtlicher Verletzungsorte erforderlich machen, was durchaus als impraktikabel bezeichnet werden kann ${ }^{79}$ und dem Charakter einheitlicher Schutzrechte entgegen laufen würde. ${ }^{80}$ Daher wurde für derartige multinationale Verletzungen eine einheitliche Anknüpfung z. B. an das Recht am gewöhnlichen Aufenthalt des Verletzers ${ }^{81}$ an das Recht des letzten Handlungsortes ${ }^{82}$ oder an das Recht des Ortes, von dem im konkreten Fall die Verhaltenssteuerung ausgegangen ist ${ }^{83}$ vorgeschlagen. ${ }^{84}$

Der Europäische Gerichtshof ${ }^{85}$ hat jüngst entschieden, dass Art. 8 Abs. 2 Rom II-VO ,dahin auszulegen ist, dass unter dem Begriff des ,Staates [...], in dem die Verletzung begangen wurde', im Sinne dieser Bestimmung der Staat zu verstehen ist, in dem das schadensbegründende Ereignis eingetreten ist. In Fällen, in denen demselben Beklagten verschiedene, in verschiedenen Mitgliedstaaten begangene Verletzungshandlungen vorgeworfen werden, ist bei der Ermittlung des schadensbegründenden Ereignisses nicht auf jede einzelne ihm vorgeworfene Verletzungshandlung abzustellen, sondern es ist eine Gesamtwürdigung seines Verhaltens vorzunehmen, um den Ort zu bestimmen, an dem die ursprüngliche Verletzungshandlung, auf die das vorgeworfene Verhalten zurückgeht, begangen worden ist oder droht.“ Mit einer derartigen Schwerpunktbildung geht freilich eine beträchtliche Rechtsunsicherheit einher. ${ }^{86}$

\footnotetext{
${ }^{76}$ Lurger/Melcher, Handbuch Internationales Privatrecht (2017) Rn. 5/86 (sowie Rn. 5/87 m.w.N., zu nach Art. 27 Rom II-VO vorrangigen Sonderkollisionsnormen).

${ }^{77}$ Womit die Anknüpfung ggf. zum Recht eines Drittstaates führen könnte, Rudolf, Europäisches Kollisionsrecht für außervertragliche Schuldverhältnisse - Rom Il-VO, ÖJZ 2010, 300 (305); für die teleologische Reduktion der Norm und das Abstellen auf den EU-Marktort MüKoBGB/Drexl, IntImmGR Rn. 140.

${ }^{78} \mathrm{MüKoBGB} /$ Drexl, IntImmGR Rn. 137, der darauf hinweist, dass sich die Vorschrift sinnvollerweise auch auf drohende Handlungen bezieht.

${ }^{79}$ BeckOK BGB/Spickhoff, VO (EG) 864/2007 Art. 8 Rn. 5.

${ }^{80}$ Krit. daher Kohler, Kollisionsrechtliche Anmerkungen zur Verordnung über die Gemeinschaftsmarke, in: FS Everling (1995) 651 (663).

${ }^{81}$ Schack, in: FS Kropholler, 651 (659 f.).

${ }^{82}$ Metzger, in: Magnus/Mankowski (eds.), European Commentaries on Private International Law Vol. 3 (2019) Art. 8 Rome II Regulation Rn. 39.

${ }^{83}$ Leible/Engel, Der Vorschlag der EG-Kommission für eine Rom II-Verordnung, EuZW 2004, 7 (13 f.).

${ }^{84}$ Die nachträgliche Wahl des Rechts eines von mehreren Handlungsortstaaten möchten Staudinger/Fezer/Koos, BGB, IntWirtschR Rn. 966, ermöglichen.

${ }^{85}$ EuGH 27.09.2017, Rs. C-24/16, Nintendo, ECLI:EU:C:2017:724 (Rn. 27 ff.).

${ }^{86}$ Mankowski, IPR II: BT ${ }^{2}$ (2019) § 2 Rn. 363. Dagegen auch Metzger, in: Drexl/Kur (Hrsg.), Intellectual Property and Private International Law (2005) 215 (221); BeckOGK/MacGuire Art. 8
} 


\section{3 (Ausschluss der) Rechtswahl}

Mit Art. 8 Abs. 3 Rom II-VO wird schließlich einem besonders strengen Verständnis der Territorialität Ausdruck verliehen. ${ }^{87}$ Mit der Bestimmung wird für außervertragliche Schuldverhältnisse aus der Verletzung von Rechten an geistigem Eigentum eine ausdrückliche Ausnahme von der Rechtswahlfreiheit gem. Art. 14 Rom II-VO angeordnet, ${ }^{88}$ womit eine parteiautonome Abweichung von der objektiven Anknüpfung nach dem Schutzlandprinzip ganz grundsätzlich ausgeschlossen wird. ${ }^{89}$

Dagegen ermöglicht in der Schweiz Art. 110 Abs. 2 IPRG den Parteien eine nachträgliche, also nach Eintritt des schädigenden Ereignisses getroffene Rechtswahl auf die schweizerische lex fori.${ }^{90}$ Freilich soll die Rechtswahl, u. a. unter Berufung auf den Wortlaut der Norm, nach wohl überwiegender Meinung allein für die Rechtsfolgen der Verletzung zulässig sein, nicht hingegen für die Anspruchsvoraussetzungen, sodass weiterhin das Schutzlandrecht zu entscheiden hat, ob das Immaterialgüterrecht überhaupt besteht und ob es verletzt ist. ${ }^{91}$

\section{Fazit}

Ein zentraler Vorteil der Schutzlandanknüpfung ist gewiss die einfache Feststellbarkeit. ${ }^{92}$ Gleichwohl sind Schutzdefizite nicht zu übersehen, ${ }^{93}$ lassen sich doch weltweite Immaterialgüterrechtsverletzungen, etwa im Bereich des Internets, auf Basis des herkömmlichen, rein territorialen Ansatzes nicht in angemessener Weise bewältigen.${ }^{94}$ Eine Verfolgung wird aufgrund des damit verbundenen Aufwands erschwert,

\footnotetext{
Rom II-VO Rn. 194 ff.

${ }^{87}$ Metzger, in: Basedow/Hopt/Zimmermann (Hrsg.), Handwörterbuch, 661 (662).

${ }^{88}$ Staudinger/Fezer/Koos, BGB, IntWirtschR Rn. 923.

${ }^{89}$ Neumayr, in: $\mathrm{KBB}^{6}$ Art. 8 Rom II-VO Rn. 5; Sonnentag, Zur Europäisierung des Internationalen außervertraglichen Schuldrechts durch die geplante Rom II-Verordnung, ZVglRWiss 105 (2006), 256 (298); krit. Schack, in: FS Kropholler, 651 (656); Richter, Parteiautonomie im Internationalen Immaterialgüterrecht (2017) 142 ff., tritt jedoch für eine primärrechtskonforme Reduktion des Rechtswahlausschlusses bei Unionsschutzrechten ein.

${ }^{90}$ Kren Kostkiewicz, IPRG/LugÜ Kommentar (2019) Art. 110 IPRG Rn. 7 f.

${ }^{91}$ Vischer, in: ZK ${ }^{2}$ Art. 136 Rn. 13; Englert, Das Immaterialgüterrecht im IPRG, in: BJM: Basler Juristische Mitteilungen (1989) 383; a.A. Jegher/Vasella, in: BaK ${ }^{3}$ Art. 110 Rn. 32; siehe auch Schack, Urheberrechtsverletzung im internationalen Privatrecht aus der Sicht des Kollisionsrechts, GRUR Int. 1985, 523 (524 f.).

${ }^{92}$ Torremans, The law applicable to copyright infringement on the Internet, NIPR 2016, 687 (692).

${ }^{93}$ Siehe bereits oben 3.2.

${ }^{94}$ Metzger, in: Basedow/Hopt/Zimmermann (Hrsg.), Handwörterbuch, 661 (662); Staudinger/Fezer/Koos, BGB, IntWirtschR Einl. Rn. 13, 34 f.
} 
teilweise sogar faktisch verhindert. ${ }^{95}$ Diesbezüglich ist eine sachgerechte Regelung dringend erforderlich. ${ }^{96}$

Wesentliche Vorschläge für die künftige Rechtsentwicklung ${ }^{97}$ beinhalten insbesondere die „ALI Principles“98 einerseits und die „CLIP Principles“99 andererseits, ${ }^{100}$ wobei erstere in Fällen ,ubiquitärer" Verletzungen von geistigen Eigentumsrechten im Internet freilich zum Recht am domicile des Rechtsinhabers tendieren ${ }^{101}$ und damit zweifellos die Interessen der US-amerikanischen Technologie- und Unterhaltungsindustrien vordringlich bedienen. ${ }^{102}$

Eine Rechtswahl schließt Art. 8 Abs. 3 Rom II-VO vollständig aus. ${ }^{103}$ Dies ist mit Blick auf den absoluten Charakter der Immaterialgüterrechte durchaus nachvollziehbar. ${ }^{104}$ In Bezug auf die hier interessierenden Rechtsfolgen einer Verletzung von geistigen Eigentumsrechten, wo die Norm den Beteiligten derzeit die Parteiautonomie entsprechend verwehrt, ${ }^{105}$ gilt dies freilich nicht in gleichem Maß, ${ }^{106}$ handelt es sich dabei doch im Grunde nicht um ein Problem des Territorialitätsprinzips. ${ }^{107}$ Vielmehr wäre de lege ferenda die Möglichkeit einer freien Rechtswahl im Verhältnis zwischen Rechtsinhaber und Verletzer in Bezug auf die Rechtsfolgen der Verletzungshandlung - etwa nach dem Vorbild von Art. 3:606 CLIP Principles - zweifelsohne erstrebenswert, könnte doch den Streitbeteiligten auf diese Weise bei den oben beschriebenen Problemen eine für beide Seiten vorteilhafte Handlungsoption eröff-

\footnotetext{
${ }^{95} \mathrm{Vgl}$. Baeumer, Anmerkungen zum Territorialitätsprinzip im internationalen Patent- und Markenrecht, in FS Fikentscher (1998) 803 (818).

${ }^{96}$ Mankowski, IPR II: BT ${ }^{2}$ (2019) § 2 Rn. 346. Krit. hinsichtlich der selbstreferenziell um sich selbst kreisenden Strukturen im Bereich des Immaterialgüterrechts Peukert, Vereinheitlichung des Immaterialgüterrechts: Strukturen, Akteure, Zwecke, RabelsZ 81 (2017) 158 (188 f.).

${ }^{97}$ Siehe auch in diesem Band Schwartze, Ausblick auf die zukünftige Entwicklung des internationalen Immaterialgüterrechts in der EU, Kap. 13.

${ }^{98}$ American Law Institute, Intellectual Property: Principles Governing Jurisdiction, Choice of Law, and Judgments in Transnational Disputes (2007).

${ }^{99}$ European Max Planck Group on Conflict of Laws in Intellectual Property, Conflict of Laws in Intellectual Property: The CLIP Principles and Commentary (2013).

${ }^{100}$ Vergleichend Treppoz, in: Nourissat/Treppoz (dir), Droit international privé et propriété intellecuelle (2010) 270.

${ }^{101} \S 321$ ALI Principles.

${ }^{102}$ Magnus/Mankowski/Metzger, Art. 8 Rome II Regulation Rn. 8; MüKoBGB/Drexl, IntImmGR Rn. 334 (,lex Hollywood“).

${ }^{103}$ Siehe oben 3.3.

${ }^{104}$ Mankowski, IPR II: BT ${ }^{2}$ (2019) § 2 Rn. 365; Heiss/Loacker, JB1 2007, 613 (637)

${ }^{105}$ Sonnentag, ZVglRWiss 105 (2006), 298; Richter, Parteiautonomie,118, 151. Die Parteien könnten ggf. danach trachten, den Streit nachträglich mit dem Abschluss von - der Rechtswahl zugänglichen - Lizenzverträgen einzufangen, NK-BGB/Grünberger, Art. 8 Rom II-VO Rn. 55.

${ }^{106}$ Leible, RIW 2008, 259; Schack, in: FS Kropholler, 656.

${ }^{107}$ Siehe zur Abgrenzung auch die Diskussion in der Schweiz zu Art. 110 Abs. 2 IPRG oben bei Fn. 91.
} 
net werden. Würde die nachträgliche Rechtswahl bei Verletzung eines Schutzrechts im genannten Parteienverhältnis zugelassen, so bliebe dadurch die Geltung des Territorialitätsgrundsatzes für die Entstehung, die Reichweite sowie die Voraussetzungen der Verletzungshandlung jedenfalls unberührt. ${ }^{108}$

${ }^{108}$ Kroll-Ludwigs, Die Rolle der Parteiautonomie im europäischen Kollisionsrecht (2013) 95.

Open Access Dieses Kapitel wird unter der Creative Commons Namensnennung 4.0 International Lizenz (http://creativecommons.org/licenses/by/4.0/deed.de) veröffentlicht, welche die Nutzung, Vervielfältigung, Bearbeitung, Verbreitung und Wiedergabe in jeglichem Medium und Format erlaubt, sofern Sie den/die ursprünglichen Autor(en) und die Quelle ordnungsgemäß nennen, einen Link zur Creative Commons Lizenz beifügen und angeben, ob Änderungen vorgenommen wurden.

Die in diesem Kapitel enthaltenen Bilder und sonstiges Drittmaterial unterliegen ebenfalls der genannten Creative Commons Lizenz, sofern sich aus der Abbildungslegende nichts anderes ergibt. Sofern das betreffende Material nicht unter der genannten Creative Commons Lizenz steht und die betreffende Handlung nicht nach gesetzlichen Vorschriften erlaubt ist, ist für die oben aufgeführten Weiterverwendungen des Materials die Einwilligung des jeweiligen Rechteinhabers einzuholen. 